\title{
ON THE NUMBER OF TERMS IN THE IRREDUCIBLE FACTORS OF A POLYNOMIAL OVER $\mathbb{Q}$
}

\author{
by A. CHOUDHRY AND A. SCHINZEL
}

(Received 29 June, 1990)

All polynomials considered in this paper belong to $\mathbb{Q}[x]$ and reducibility means reducibility over $\mathbb{Q}$. It has been established by one of us [5] that every binomial in $\mathbb{Q}[x]$ has an irreducible factor which is either a binomial or a trinomial. He has further raised the question "Does there exist an absolute constant $K$ such that every trinomial in $\mathbb{Q}[x]$ has a factor irreducible over $\mathbb{Q}$ which has at most $K$ terms (i.e. $K$ non-zero coefficients)?"

A similar question could be asked for a quadrinomial, or, more generally, for a polynomial with $m$ non-zero coefficients. This paper deals with the general problem, that could be formulated as follows:

Given a positive integer $m$ does there exist a number $K$ such that every polynomial in $\mathbb{Q}[x]$ with $m$ non-zero coefficients has a factor irreducible over $\mathbb{Q}$ with at most $K$ non-zero coefficients ( $K$ "terms")?

If for a given $m$ numbers $K$ with the above property exist we denote by $K(m)$ the least of them, otherwise we put $K(m)=\infty$.

We shall prove

THEOREM. (i) $K(3) \geq 8$, (ii) $K(4) \geq 13$, (iii) $K(5) \geq 14$, (iv) $K(6) \geq 16$ and for every $m>2: K(m)>\max \left\{2 m, c_{1} m^{c_{2}}\right\}$, where $c_{1}=0.014$ and $c_{2}=1.22$ are both independent of $m$.

Proof.

(i) $m=3$.

This case has been dealt earlier by Bremner [1] who has proved $K(3) \geq 8$, without, however explicitly giving the trinomial concerned. We shall obtain the same result by a numerical example. We write

$$
f(x)=x^{7}+20 x^{6}+200 x^{5}+2450 x^{4}+29000 x^{3}+545000 x^{2}+8101250 x+35275000 .
$$

Then we have the identity

$$
f(x) f(-x)=-x^{14}-27180501562500 x^{2}+35275000^{2} .
$$

We prove the irreducibility of $f(x)$ and $f(-x)$ by using the method of G. Dumas [3] (cf. Dorwart [2]) based on Newton polygon. The Newton polygon corresponding to $f(x)$ for the prime 2 is shown on Figure 1.

It follows from the irreducibility theorem of Dumas that the proper factors of $f(x)$ can only be of degree 1 or 6 , thus if $f(x)$ is reducible it has a factor $x-\lambda$, where $\lambda$ is an integer. We must have $\lambda \mid 35275000$, also $10 \mid \lambda$ and finitely many possible values of $\lambda$ are easily ruled out. Therefore $f(x)$ is irreducible and as it has 8 terms $K(3) \geq 8$.

(ii) $m=4$. Let

$$
\begin{aligned}
f(x)= & x^{12}+4 x^{11}+8 x^{10}+16 x^{9}+32 x^{8}+64 x^{7}+128 x^{6}+192 x^{5}+256 x^{4} \\
& +384 x^{3}+512 x^{2}+640 x+512 .
\end{aligned}
$$




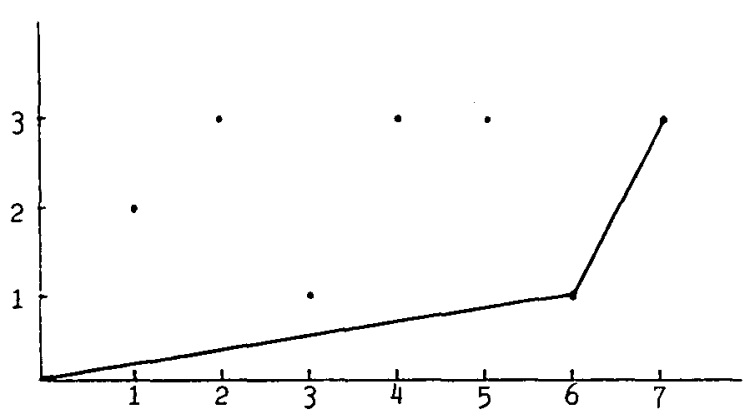

Figure 1.

We have the identity

$$
f(x) f(-x)=x^{24}+32768 x^{4}+114688 x^{2}+262144 .
$$

To prove that $f(x)$ is irreducible, we construct the Newton polygon corresponding to $f(x)$ for the prime 2 (Figure 2).

It then follows from the irreducibility theorem of Dumas that the proper factors of $f(x)$ must be of degree 1 or 11 . Thus if $f(x)$ is reducible it must have a factor $x-\lambda$, where $\lambda$ is an integer and $\lambda \mid 512$. All such factors are easily ruled out. Hence $f(x)$ is irreducible and as it has 13 non-zero coefficients $K(4) \geq 13$.

(iii) $m=5$. Let

$$
\begin{aligned}
f(x, t)= & 8 x^{13}-16 x^{12}+16 x^{11}-16 x^{10}+16 x^{9}-16 x^{8}+16 x^{7}-8 t x^{6} \\
& +(16 t-16) x^{5}-(20 t-24) x^{4}+(24 t-32) x^{3}-(27 t-38) x^{2} \\
& +(30 t-44) x+2 t^{2}-23 t+30 \\
= & 2 t^{2}-t\left(8 x^{6}-16 x^{5}+20 x^{4}-24 x^{3}+27 x^{2}-30 x+23\right)+8 x^{13}-16 x^{12}+16 x^{11} \\
& -16 x^{10}+16 x^{9}-16 x^{8}+16 x^{7}-16 x^{5}+24 x^{4}-32 x^{3}+38 x^{2}-44 x+30 .
\end{aligned}
$$

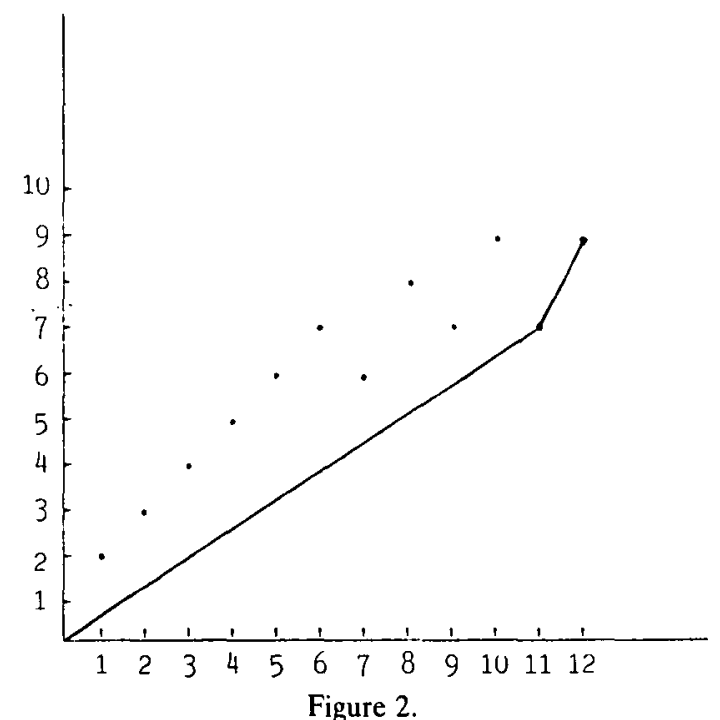


As the coefficients of $t^{2}, t, t^{0}$ have the highest common factor $1, f(x, t)$ has no factors depending only on $x$. Moreover its discriminant with respect to $t$ is not a perfect square and $f(x, t)$ is thus irreducible in $x, t$. By virtue of Hilbert's irreducibility theorem ([4], cf. also [6], p. 179) there exist infinitely many integers $t$ for which $f(x, t)$ is irreducible. The identity

$$
\begin{aligned}
f(x, t) f(-x, t)= & -64 x^{26}-\left(32 t^{3}+88 t^{2}-608 t+608\right) x^{6} \\
& -\left(80 t^{3}-305 t^{2}+324 t-68\right) x^{4} \\
& -\left(108 t^{3}-494 t^{2}+728 t-344\right) x^{2}+\left(2 t^{2}-23 t+30\right)^{2}
\end{aligned}
$$

provides us with infinitely many examples which show that

$$
K(5) \geq 14 \text {. }
$$

(iv) $m=6$.

To prove $K(6) \geq 16$, we use another polynomial defined by

$$
\begin{aligned}
f(x, t)= & (575-2 t) x^{15}+\left(2 t^{3}-444 t^{2}+30032 t-582402\right) x^{14} \\
& +\left(t^{3}-222 t^{2}+15016 t-291070\right) x^{13}+\left(-2 t^{2}+304 t-7708\right) x^{12} \\
& +\left(-t^{2}+152 t-3812\right) x^{11}+(4 t-28) x^{10}+2 t x^{9}+74 x^{8} \\
& +42 x^{7}+20 x^{6}+12 x^{5}+6 x^{4}+4 x^{3}+2 x^{2}+2 x+1 \\
= & \left(2 x^{14}+x^{13}\right) t^{3}-\left(444 x^{14}+222 x^{13}+2 x^{12}+x^{11}\right) t^{2} \\
& -\left(2 x^{15}-30032 x^{14}-15016 x^{13}-304 x^{12}-152 x^{11}-4 x^{10}-2 x^{9}\right) t \\
& +575 x^{15}-582402 x^{14}-291070 x^{13}-7708 x^{12}-3812 x^{11} \\
& -28 x^{10}+74 x^{8}+42 x^{7}+20 x^{6}+12 x^{5}+6 x^{4}+4 x^{3}+2 x^{2}+2 x+1 .
\end{aligned}
$$

The polynomial $f(x, t)$ is irreducible as a polynomial in two variables. Indeed, it has no factor depending only on $x$, since the coefficients of $t^{3}$ and $t^{0}$ are relatively prime. Therefore the only possible factorisation would be

$$
f(x, t)=\{a(x) t+b(x)\}\left\{c(x) t^{2}+d(x) t+e(x)\right\} .
$$

Hence

(i) $a(x) c(x)=2 x^{14}+x^{13}$

(ii) $a(x) d(x)+b(x) c(x)=-444 x^{14}-222 x^{13}-2 x^{12}-x^{11}$

(iii) $a(x) e(x)+b(x) d(x)=-2 x^{15}+\ldots+2 x^{9}$

(iv) $b(x) e(x)=f(x, 0)$

Let $a, b, c, d, e$ be divisible exactly by $x^{\alpha}, x^{\beta}, x^{\gamma}, x^{\delta}, x^{\epsilon}$ respectively. By (i) $\alpha+\gamma=$ 13 , by (iv) $\beta=\epsilon=0$, hence by (iii) either $\alpha \geq 9, \delta \geq 9$ or $\alpha=\delta$. In the former case, the degrees with respect to $x$ of both factors of the first term on the left hand side of (ii) are at least 9 , hence the degree of the product is at least 18 , a contradiction. In the latter case by (ii) either $\alpha+\delta=2 \alpha \geq 11, \beta+\gamma=13-\alpha \geq 11$, hence $26=2 \alpha+2(13-\alpha) \geq 33$, a contradiction, or $\alpha+\delta=\beta+\gamma ; 2 \alpha=13-\alpha, 3 \alpha=13$, a contradiction.

Since $f(x, t)$ is irreducible as a polynomial in $x$ and $t$, Hilbert's theorem gives the existence of infinitely many integers such that $f(x, t)$ is irreducible in $x$. We also note that in the product $f(x, t) f(-x, t)$ only the coefficients of $x^{30}, x^{28}, x^{26}, x^{24}, x^{22}$ and $x^{0}$ are not 
zero. The other coefficients are all 0 , so we have only six non-zero terms in the product and it follows that

$$
K(6) \geq 16 \text {. }
$$

(v) For the general case of a polynomial with $m$ non-zero terms, we establish $K(m) \geq 2 m$ by an explicit example.

Let

$$
\begin{aligned}
f(x)= & p x^{2 m-1}+2 p x^{2 m-2}+2 p x^{2 m-3}+\ldots+2 p x^{3} \\
& +p^{2} x^{2}+2 p(p-1) x+2(p-1)^{2} .
\end{aligned}
$$

Then the only non-zero terms in the product $f(x) f(-x)$ are the coefficients of $x^{4 m-2}$, $x^{2 m-2}, x^{2 m-4}, x^{2 m-6}, \ldots, x^{4}$ and $x^{0}$. Thus, the product $f(x) f(-x)$ has only $m$ non-zero terms and if we take $p$ as an odd prime, both $f(x)$ and $f(-x)$ are irreducible in view of Einstenstein's criterion.

This proves that

$$
K(m) \geq 2 m \text {. }
$$

To prove $K(m)>c_{1} m^{c_{2}}$, we use a result of Verdenius [7] who has established that for every positive integer $n$, there exists a polynomial $f(x)$ of the $n^{\text {th }}$ degree with real integer coefficients such that $f^{2}(x)$ consists of less than $\frac{1}{5}\left(162 n^{\log _{9} 6}-12\right)$ terms. For any such polynomial $f(x)$, we have the identity

$$
\{f(p x)-p f(x)\}\{f(p x)+p f(x)\}=f^{2}(p x)-p^{2} f^{2}(x) .
$$

While the two factors on the left hand side have $n$ and $(n+1)$ terms respectively, their product has $m$ terms, $m<\frac{1}{5}\left(162 n^{\log _{9} 6}-12\right)$. Also, if $f(x)=a_{0} x^{n}+\ldots+a_{n}$ and $p$ is a prime number such that $p+a_{0}$ and $p+a_{n}$, both factors on the left hand side are irreducible in view of Einsenstein's criterion.

It follows that $K(m) \geq n$.

Now

yields

$$
m<\frac{1}{5}\left(162 n^{\log _{9} 6}-12\right)
$$

$$
n>\left(\frac{5 m+12}{162}\right)^{\log _{6} 9}
$$

or

where

$$
n>c_{1} m^{c_{2}}
$$

$$
\begin{aligned}
& c_{1}=0 \cdot 014 \ldots \\
& c_{2}=1 \cdot 22 \ldots
\end{aligned}
$$

Hence for every integer $m, K(m) \geq c_{1} m^{c_{2}}$.

Thus, for each $m, K(m) \geq 2 m$ and also $K(m)>c_{1} m^{c_{2}}$, so we have

$$
K(m) \geq \max \left\{2 m, c_{1} m^{c_{2}}\right\}
$$

where $c_{1}=0 \cdot 014 \ldots$ and $c_{2}=1 \cdot 22 \ldots$. 


\section{REFERENCES}

1. A. Bremner, On reducibility of trinomials, Glasgow Math. J. 22 (1981), 155-156.

2. H. L. Dorwart, Irreducibility of polynomials, Amer. Math. Monthly 42 (1935), 369-381.

3. G. Dumas, Sur quelques cas d'irréductibilité des polynômes à coefficients rationels, Journal Math. pures appl (2) 6 (1906) 191-258.

4. D. Hilbert, Über die Irreduzibilität ganzer rationaler Funktionen mit ganzzahligen Koeffizienten, Ges. Abh. Bd II 264-286. 63-70.

5. A. Schinzel, Some unsolved problems in polynomials, Matematicka biblioteka, 25 (1963)

6. A. Schinzel, Selected Topics on Polynomials, (The University of Michgan Press, 1982).

7. W. Verdenius, On the number of terms of the square and the cube of polynomials, Indag. Math. 11 (1949) 459-465.

\author{
Deputy High Commissioner, \\ High COMMISSION OF INDIA, \\ 31 Grange RoAd, \\ SINGAPORE 0923.
}

\author{
Mathematical Institute, \\ Polish ACademy of Sciences, \\ P.O. Box 137, 00950 Warszawa, \\ Poland.
}

